Revista Electrónica Complutense de Investigación en Educación Musical ISSN-e: 1698-7454

http://dx.doi.org/10.5209/RECIEM.49527

\title{
Integración de la especialidad de flamenco en el grado superior de música
}

\author{
Carlos Pacheco Torres ${ }^{1}$; Rosario Ortega-Ruz ${ }^{2}$; Antonio J. Rodríguez Hidalgo ${ }^{3}$
}

Recibido: 06 de septiembre de 2016 / Aceptado: 31 de enero de 2017

Resumen. Se presenta una experiencia didáctica desarrollada en un Conservatorio Superior de Música que integra alumnado de especialidades sinfónicas -tradición clásica- y de tradición flamenca, mediante la práctica orquestal flamenca. Se ejemplifica la secuencia didáctica y una investigación sobre la autopercepción del alumnado sobre su propio aprendizaje. 47 estudiantes cumplimentaron un autoinforme. Los resultados indican que el alumnado de ambas tradiciones valora favorablemente los aspectos que les resultan novedosos. Los resultados de la experiencia y de la investigación se discuten en relación a las bases de aprendizaje colaborativo y significativo.

Palabras clave: flamenco; música clásica; aprendizaje colaborativo; aprendizaje significativo; compás flamenco; bulería por soleá.

\section{[en] Integration of the specialty of flamenco in the superior degree of music}

Abstract. A learning experience developed in a Conservatory of Music that integrates both students of classical and flamenco tradition by means of the flamenco orchestral practice is presented. The teaching sequence and a research about the self-perception of students on their learning are exemplified. 47 students completed a self-report. The results indicate that students of both traditions favorably value the aspects that are novel to them. The results of both the experience and the research are discussed in relation to the bases of collaborative and meaningful learning.

Keywords: flamenco tradition; classic tradition; collaborative learning; meaningful learning; flamenco beat; Bulería por soleá.

Sumario. 1. Introducción. 2. Contexto de la experiencia didáctica y de la investigación. 3. Marco teórico. 4. Objetivos de la experiencia didáctica. 5. Experiencia didáctica. 6. Metodología de la investigación. 7. Resultados. 8. Discusión y conclusiones. 9. Referencias bibliográficas.

Cómo citar: Pacheco Torres, C.; Ortega-Ruz, R.; Rodríguez Hidalgo, A.J. (2017). Integración de la especialidad de flamenco en el grado superior de música, en Revista Electrónica Complutense de Investigación en Educación Musical, 14, 351-368.

\footnotetext{
1 Conservatorio Superior de Música de Córdoba

61carlosp@gmail.com

2 Universidad de Córdoba

ortegaruiz@uco.es

$3 \quad$ Universidad de Córdoba

ajrodriguez@uco.es
} 


\section{Introducción}

La pedagogía musical, partiendo de la base de los enfoques cognitivistas/constructivistas, en los últimos años muestra una tendencia a ampliarse mediante enfoques orientados a la educación integral del alumnado. Son muchos los autores que han planteado fundamentos en este sentido. Por ejemplo, Leiva y Mates (1998) y Ramírez (2006) sostienen que la experiencia musical permite experimentar sentimientos y emociones que ayudan a reforzar en el alumnado el espíritu crítico, el respeto hacia uno mismo y hacia los demás, la confianza en sí mismo, entre otros aspectos. Los procesos de enseñanza y aprendizaje limitados exclusivamente al intelecto parecen resultar insuficientes, haciéndose necesario abordar las dimensiones afectiva y de relación interpersonal. En este sentido, los procesos de aprendizaje en la enseñanza superior de música en los conservatorios no deberían ser ajenos a esta visión.

El lenguaje musical flamenco, representado en los diferentes estilos o "palos" estructurados para guitarra flamenca de concierto, posee elementos constitutivos que permiten su análisis y aplicación desde la teoría musical general y desde la teoría específica del lenguaje musical flamenco. Dichos elementos cobran una nueva dimensión si se analizan y aplican en el contexto educativo.

La práctica orquestal flamenca nos permite desarrollar una propuesta creativa a la par que didáctica. Creativa por cuanto es necesario diseñar herramientas didácticas que permitan llevar los elementos presentes en la guitarra flamenca hacia un formato orquestal. Didáctica porque estas herramientas son aplicadas en el aula, ayudándonos a comprender la interacción entre iguales.

En este sentido, la dimensión didáctica juega un papel fundamental para entender la presencia del flamenco como fenómeno integrador en el proceso de enseñanza y aprendizaje en los dos perfiles de alumnado que se dan en el Conservatorio Superior de Música de Córdoba en las asignaturas de Conjunto Instrumental Flamenco y Ensemble Flamenco. Por un lado, encontramos alumnado que proviene de una prueba de acceso, muy familiarizado con los estilos flamencos, pero poco con el lenguaje musical -serán los que llamaremos de tradición flamenca, que se sienten más cómodos con la transmisión oral del conocimiento-, mientras que el resto de alumnos/as son egresados del Conservatorio Profesional - que en adelante llamaremos alumnado de tradición clásica, que dominan el lenguaje musical-.

A continuación se presenta una experiencia didáctica que integra alumnado de especialidades sinfónicas - tradición clásica- y de tradición flamenca, mediante la práctica orquestal flamenca, partiendo de la revisión del contexto y de los fundamentos teóricos. Posteriormente se expone una pequeña investigación sobre la autopercepción del alumnado sobre su propio aprendizaje en dicha experiencia.

\section{Contexto de la experiencia didáctica y de la investigación}

Las enseñanzas de flamenco en Grado Superior de Música en Andalucía se materializan con la creación de la Especialidad de Flamenco ${ }^{4}$. Dicha especialidad tiene como único centro de referencia en Andalucía el Conservatorio Superior de Música

Real Decreto 707/2011, de 20 de mayo, por el que se crea la especialidad de Flamenco en las enseñanzas artísticas superiores de Grado de Música y se regula su contenido (BOE de 9 de junio de 2011). 
"Rafael Orozco" de Córdoba. Esta especialidad tiene tres itinerarios: Guitarra Flamenca, Flamencología y Cante Flamenco.

En el plan de estudios de la Especialidad de Flamenco se imparte la materia Conjunto Instrumental Flamenco. Esta materia es de carácter obligado para el alumnado de la especialidad de flamenco. En esta asignatura, el alumnado de dicha especialidad comparte estudio con alumnado de otros instrumentos de tradición clásica, ya que también se les oferta esta materia como optativa. Así, en el aula de la materia Conjunto Instrumental Flamenco confluyen dos perfiles de alumnado claramente distintos: los de tradición flamenca (guitarra flamenca, flamencología y cante flamenco) y los de otras especialidades de tradición clásica (violín, viola, violonchelo, contrabajo, flauta, clarinete, percusión, entre otras). Tanto uno como otro alumnado han experimentado procesos de aprendizaje sensiblemente diferentes y han desarrollado esquemas cognitivos distintos en materia musical. A priori, parten de conocimientos previos diferentes, marcados en buena medida por el predominio de experiencias diferentes, fundamentalmente de carácter oral y de oído en el caso del alumnado de música flamenca, versus experiencias basadas en el uso y dominio del lenguaje musical en el caso del alumnado de música clásica. De la confluencia de estos dos perfiles de alumnado surge el reto de elaborar estrategias didácticas que posibiliten nuevos aprendizajes colaborativos y significativos para ambos. De aquí se desprende la necesidad de conocer, comprender e integrar el lenguaje musical flamenco en la práctica orquestal. Ello supone la posibilidad de que alumnado de las dos tradiciones pueda interactuar y aprender entre sí.

Para dar respuesta a esta necesidad educativa, hemos desarrollado un trabajo en el que se ha combinado el diseño e intervención didáctica y la investigación en el aula. En este trabajo, el profesor de la asignatura es, a la vez, el creador de una herramienta didáctica y aplicador de la misma en el aula, e investigador sobre este ejercicio de innovación.

\section{Marco teórico}

La temática de nuestra investigación parte de la reflexión en el aula referida a la incorporación de una nueva especialidad, la cual posee sus propios rasgos de singularidad. En la revisión de la bibliografía existente no se encontraron artículos similares que aborden específicamente experiencias relacionadas con la práctica orquestal de la música flamenca en el aula. Sin embargo, podemos encontrar teorías y trabajos que nos permitan reforzar la opción metodológica elegida para llevarla a cabo.

Tanto el trabajo en el aula como la aplicación de las herramientas didácticas que se emplean se realizan sobre la base de la investigación acción en la cual, a parte de otras características, se proyecta fundamentalmente la doble labor del docente asumiendo los roles de profesor e investigador (Stenhouse, 2003; Elliott, 1994; Schön, 1987; Carr y Kemmis, 1986). También, la proyección de ésta teoría hacia todo lo que supone el trabajo colaborativo (Johnson y Johnson, 1999). De igual forma, el conocimiento en relación a la interacción e intercambio de códigos entre alumnado de tradición clásica y flamenca y la percepción que tienen de su propio aprendizaje se fundamenta sobre la base del aprendizaje significativo (Ausubel, 1963,1976; Rivera Muñoz, 2004; Rusinek, 2004). 
Josep María Vilar, en su artículo titulado Mesa redonda: "Investigación aplicada -investigación-acción y currículo oculto en la enseñanza obligatoria"- ${ }^{5}$, aborda la temática del currículo y su desarrollo desde los conservatorios de música. Su temática está centrada en lo que es y debe ser la música, desde la perspectiva del alumnado, el profesorado, el objeto de aprendizaje, así como los componentes sociales que lo condicionan en el aula. Analiza con sentido crítico el posicionamiento epistemológico tradicional presente en los conservatorios, introduciendo el concepto de currículo oculto. La actividad propia de un conservatorio no debe de ser la música, sino la pedagogía de la música. Para Vila, el currículo oculto presente en los conservatorios actúa como un lastre en la búsqueda de otras vías válidas para la investigación.

"El currículo oculto, en su priorización del acto interpretativo, relega en segundo plano actitudes más reflexivas que deben estar en la base de cualquier investigación. Es el currículo oculto el que permite seguir ignorando otras culturas musicales". (Vilar, 2000, p. 6)

Considerando lo anterior, Vila apunta también hacia la necesidad de desarrollar nuevas investigaciones desde los conservatorios que ayuden a superar el denominado currículo oculto.

\section{Objetivos de la experiencia didáctica y de la investigación}

El trabajo desarrollado se ha orientado a alcanzar los siguientes objetivos didácticos y de investigación:

1) Crear secuencias didácticas que nos permitan llevar los elementos de la guitarra flamenca hacia un formato orquestal (objetivo didáctico).

2) Aplicar las secuencias didácticas específicas para cada estilo flamenco en el aula (objetivo didáctico).

3) Conocer la percepción del alumnado respecto a su propio aprendizaje (objetivo de investigación).

4) Conocer si se cumplen las condiciones para que se produzca un aprendizaje significativo y colaborativo mediante la práctica orquestal flamenca (objetivo de investigación).

\section{Experiencia didáctica}

\subsection{Diseño didáctico}

La metodología didáctica utilizada para el diseño y para el desarrollo de la experiencia ha seguido los presupuestos anteriormente expuestos de la investigación-acción colaborativa (Johnson y Johnson, 1999; Stenhouse, 2003), así como del aprendizaje significativo (Ausubel, 1983,1976; Rusinek, 2004). Se ha optado por asumir esta metodología didáctica con la finalidad de mejorar la práctica educativa.

Trabajo presentado en las I jornadas de investigación en educación musical (Ceuta 1-3 de octubre de 1998). 
Desde una perspectiva socio-constructivista del aprendizaje se entiende que, partiendo de las ideas y conocimientos previos del alumnado, con la orientación y propuesta del profesor-investigador que estimula la integración colaborativa de alumnado con diversos perfiles, se podrán producir aprendizajes bien anclados y altamente significativos. La investigación-acción permitirá también al docente una retroalimentación continua beneficiosa, tanto para la optimización de los procesos de enseñanza-aprendizaje, como para generar innovación educativa. En este proceso continuo de retroalimentación tendrá un importante valor estratégico la recogida de información (evaluación inicial, continua y final) mediante los siguientes instrumentos:

1. Cuestionarios.

2. Grabaciones audiovisuales.

3. Entrevistas semiestructuradas a profesores tutores de las diferentes especialidades instrumentales.

4. Notas de clase.

El material didáctico se ha confeccionado partiendo de la creación de secuencias didácticas específicas para cada estilo flamenco, encaminadas a elaborar un proceso de ampliación instrumental desde la guitarra flamenca hacia la orquesta.

- La organización del trabajo en el aula se ha realizado de la siguiente forma:

- Las sesiones de cada clase han sido de una hora y media a la semana, distribuidas en dos grupos ( $11.30 \mathrm{~h}$ a $13.00 \mathrm{~h}$ y $13.00 \mathrm{~h}$ a $14.30 \mathrm{~h}$ ), en los cuales se incluían alumnado de diferentes perfiles.

Para cada sesión de clase se estructuraron dos grupos que, a su vez, se han dividido en subgrupos. El siguiente esquema lo representa:

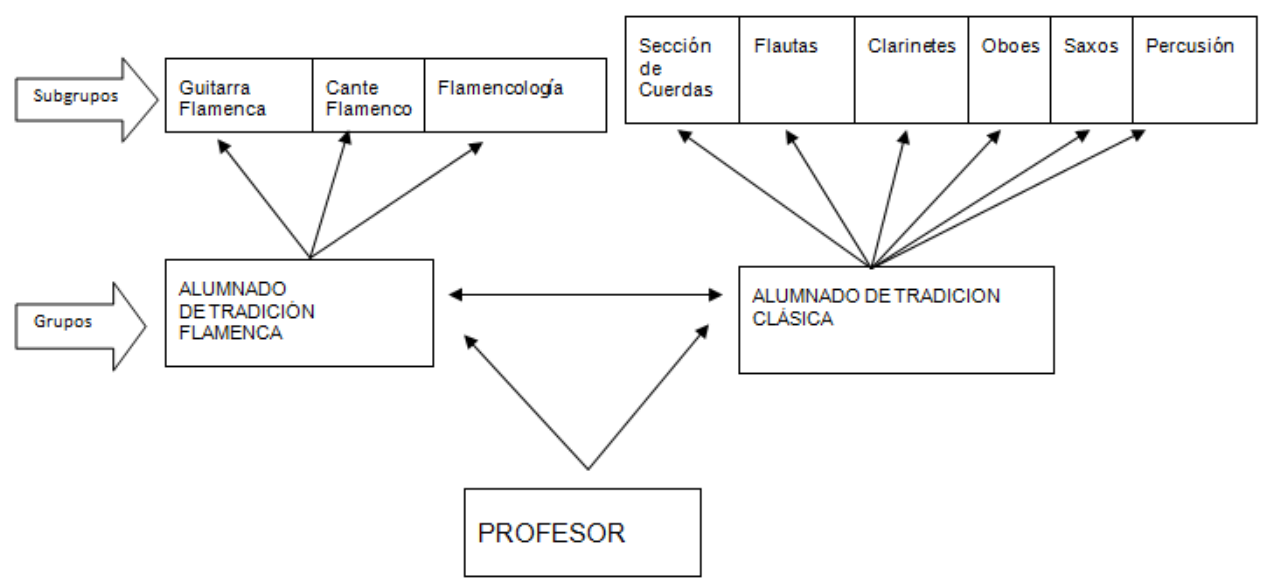

Figura 1. Estructura del trabajo en el aula durante las clases de Conjunto Instrumental Flamenco y Ensemble Flamenco. 
- Las TIC también han formado parte en la organización del grupo clase. Mediante la aplicación para "smartphone" de mensajería instantánea "Whatsapp", el profesor ha estructurado una comunicación rápida y eficaz creando un grupo de "Whatsapp" para cada subgrupo de instrumentos. De esta manera, la autoevaluación del profesor, así como las observaciones de los aspectos positivos y a mejorar del alumnado tras cada sesión, pueden comunicarse durante la semana con el objetivo de reforzar, entre otros aspectos, la autoevaluación tanto del profesor como de los alumnos, así como destacar en cada semana la importancia que tiene el trabajo y compromiso individual en el resultado final.

- Cada diez sesiones de clase se realiza un encuentro con los dos grupos, que es grabado para su posterior análisis e interpretación.

- En cada encuentro se invita a diferentes tutores, tanto de alumnado de tradición clásica como de tradición flamenca.

- Se representan las obras en concierto final en el auditorio del centro. La presencia de público especializado en la materia, tanto de tradición flamenca como clásica, confiere una significatividad especial a un momento que marca la finalización del proceso de cada obra.

\subsection{Desarrollo de la experiencia didáctica}

La experiencia se ha llevado a cabo con alumnado de la asignatura de Conjunto Instrumental Flamenco (especialidad de Flamenco) y Ensemble Flamenco (especialidades instrumentales de tradición clásica) en el Conservatorio Superior de Música de Córdoba, durante los cursos académicos 2012-13, 2013-14, 2014-15 y 2015-16.

En primer lugar, a partir de la ampliación instrumental realizada para cada estilo flamenco, se diseña una secuencia didáctica que permita introducir al alumnado de tradición clásica en las características musicales fundamentales presentes en el estilo flamenco. De igual forma, el alumnado de tradición flamenca ayudará en la retroalimentación de conocimientos con el alumnado de tradición clásica desde una actitud constructiva y colaborativa. Dicha secuencia didáctica se considera fundamental como paso previo de la práctica orquestal de la obra en su totalidad.

Por motivos de espacio, aplicaremos la secuencia didáctica solo en el estilo de bulería por soleá, entendiendo que se proyecta de igual forma a los otros estilos flamencos seleccionados. Las etapas en las que se divide la citada secuencia se describen a continuación:

\subsubsection{Explicación rítmica y armónica del estilo}

Se explican los elementos musicales presentes en el estilo de bulería por soleá, desde la transmisión oral, fundamentalmente los referidos a su rítmica y armonía, para de esta forma intentar llegar a una transcripción del compás lo más cercano al óptimo en lo que se refiere a la aplicación en un formato orquestal final, es decir, para que, tras la ampliación instrumental, el resultado, ya en formato orquestal, siga sonando "flamenco". 


\section{Explicación rítmica:}

Es importante que los alumnos de tradición clásica comprendan cómo conciben el compás de bulería por soleá los músicos de tradición flamenca. Los flamencos lo miden de la siguiente forma, enfatizando los acentos en los tiempos: 3, 6, 8, 10 y 12 .

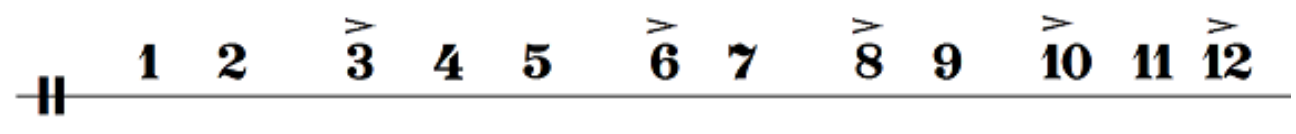

\section{Ejemplo $\mathbf{N}^{0} 1$}

Lo anterior nos deja claro dónde se encuentran ubicados los tiempos fuertes o acentuaciones más importantes del estilo. Establecer cómo llevar esta medida a la partitura es fundamental para un óptimo resultado de la práctica orquestal flamenca en el aula.

A continuación, trabajaremos varias opciones de clasificación del compás, determinando en la práctica orquestal cuál de ellas resulta ser la más conveniente.

\section{Opción A:}

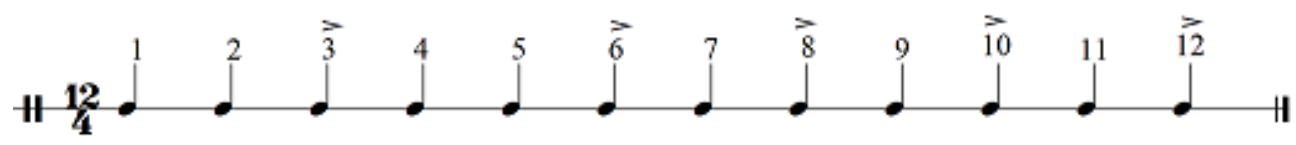

\section{Ejemplo $\mathbf{N}^{0} 2$}

El aspecto positivo de esta opción radica en que podemos situar los 12 tiempos flamencos en un solo compás. Esto no presenta grandes complicaciones para el alumnado de tradición flamenca, pues en todo momento están sintiendo los acentos importantes (aspecto que ellos tienen interiorizado desde la transmisión oral).

La dificultad de esta opción afecta fundamentalmente al alumnado de tradición clásica ya que, para ellos, por regla general, el acento fuerte está en la primera parte del compás y el tiempo débil en la última. En esta opción ven todo lo contrario; el tiempo 1 como débil y el último (12) como fuerte. Además, los acentos se encuentran ubicados irregularmente. Esto hace perder naturalidad y fluidez en la lectura. Creemos, por tanto, que es una opción poco válida, al menos en el ámbito de la práctica orquestal flamenca.

\section{Opción B:}

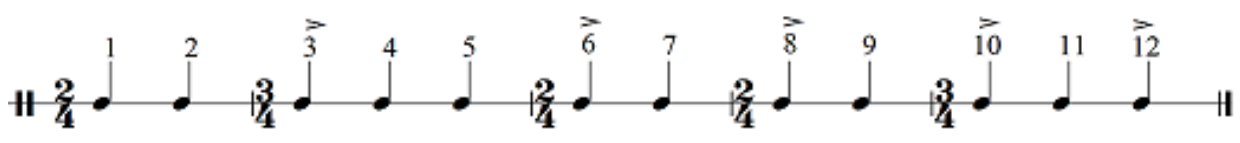




\section{Ejemplo $\mathbf{N}^{0} 3$}

Aquí nos acercamos bastante más al ritmo deseado. Ahora el compás flamenco de 12 tiempos se transcribe como la amalgama $2+3+2+2+3$. El único inconveniente para el alumnado de tradición clásica, el cual no conoce ni ha experimentado este estilo flamenco, es que ve en un compás (el último de la amalgama) acentos importantes del estilo, en la primera y tercera parte. Esto produce una desconcentración en la lectura del alumnado de tradición clásica, teniendo que estar marcando siempre este acento en la última parte del compás. Si consideramos, entre otros factores, los contratiempos que llevan las palmas y su relación con la guitarra, nos encontramos con los mismos inconvenientes de la opción anterior.

\section{Opción C:}

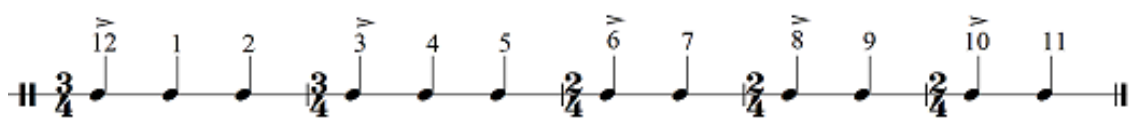

\section{Ejemplo $\mathbf{N}^{\circ} 4$}

Esta opción ha resultado ser la más beneficiosa, ya que en ella se solucionan los inconvenientes encontrados en las dos anteriores. En su relación con el cante y las palmas, el tiempo 12 ejerce un protagonismo muy especial. Así, el tiempo 10 marca el cierre del compás y el tiempo 12 cumple dos funciones: la de reafirmar el final y, a su vez, marcar el inicio del compás siguiente. Ahora los alumnos/as de tradición clásica están viendo y sintiendo los acentos importantes del estilo en la primera parte de cada compás de la amalgama, que se define como $3+3+2+2+2$, coincidiendo con el alumnado de tradición flamenca a la hora de marcar el compás y enfatizar sus acentuaciones.

Las palmas marcan el compás con un ciclo rítmico que les es característico.

Una vez definida la amalgama veamos como interactúa con las palmas:

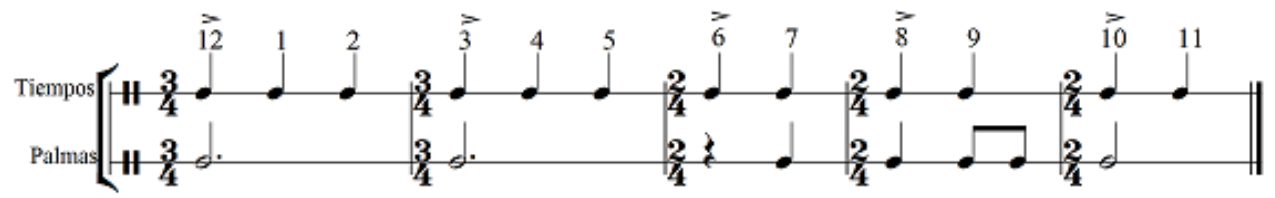

\section{Ejemplo $\mathbf{N}^{\circ} 5$}

Las palmas enfatizan los tiempos 12, 3, 8 y 10. Dejan el tiempo 6 libre, pero las negras y corcheas presentes en los tiempos 7,8 y 9 , respectivamente, ayudan a marcar con mayor énfasis los tiempos 8 y 10.

Experiencia compartida.

En este momento de la experiencia, mientras el alumnado de tradición flamenca ejecuta sin partitura, es decir, mostrando y compartiendo sus experiencias vividas desde la tradición oral o, dicho en su lenguaje, "tocan por bulería por soleá", se le 
pide al alumnado de tradición clásica que ejecute una polirritmia; es decir, que marquen los tiempos con el pie al tiempo que ejecutan las palmas (Ejemplo $\mathrm{N}^{\mathrm{o}} 5$ ).

\section{Explicación armónica.}

La cadencia característica se desenvuelve en el modo de La flamenco entre los grados I, II y III.6 El segundo grado ejerce el carácter de dominante, siendo el grado que constantemente va resolviendo al primer grado. En la siguiente transcripción, veremos cómo interactúan el compás, las palmas y la guitarra flamenca. Se trata de un ciclo de dos compases flamencos $(3+3+2+2+2)$ que se repiten las veces que se determine. Finalmente, la segunda terminación indica el cierre del compás.
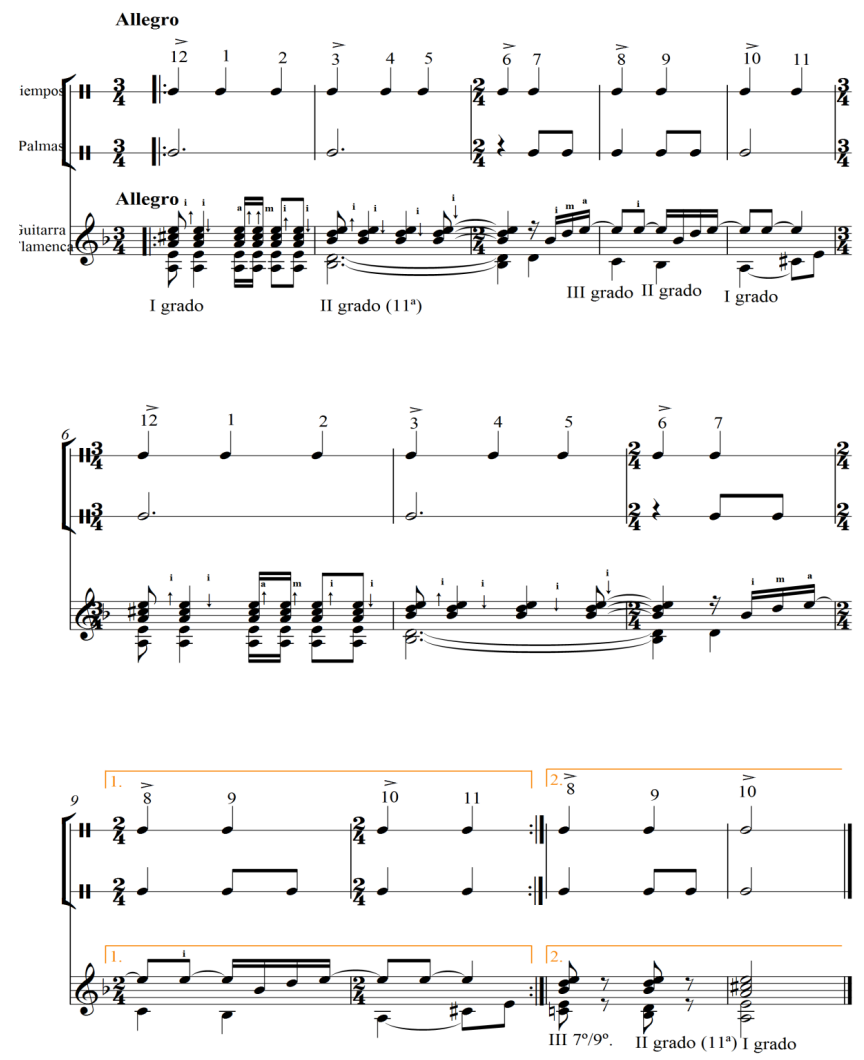

\section{Ejemplo $\mathbf{N}^{0} 6$}

\subsubsection{Práctica en conjunto de la cadencia característica del estilo}

Una vez asimilados el ritmo y la armonía, se incorporan los instrumentos de tradición clásica en una primera toma de contacto con la cadencia característica del estilo. En una escritura a cuatro voces, se determina una voz para cada grupo de instrumentos:

Una extensa y rigurosa explicación sobre el modo flamenco lo podemos encontrar en los artículos realizados por profesor Manuel Cera Vera. 
- Soprano: violines y flautas.

- Contralto: oboes y clarinetes.

- Tenor: chelo y fagot.

- Bajo. contrabajo.

Al mismo tiempo, el alumnado de tradición flamenca interactúa interpretando la guitarra y las palmas.
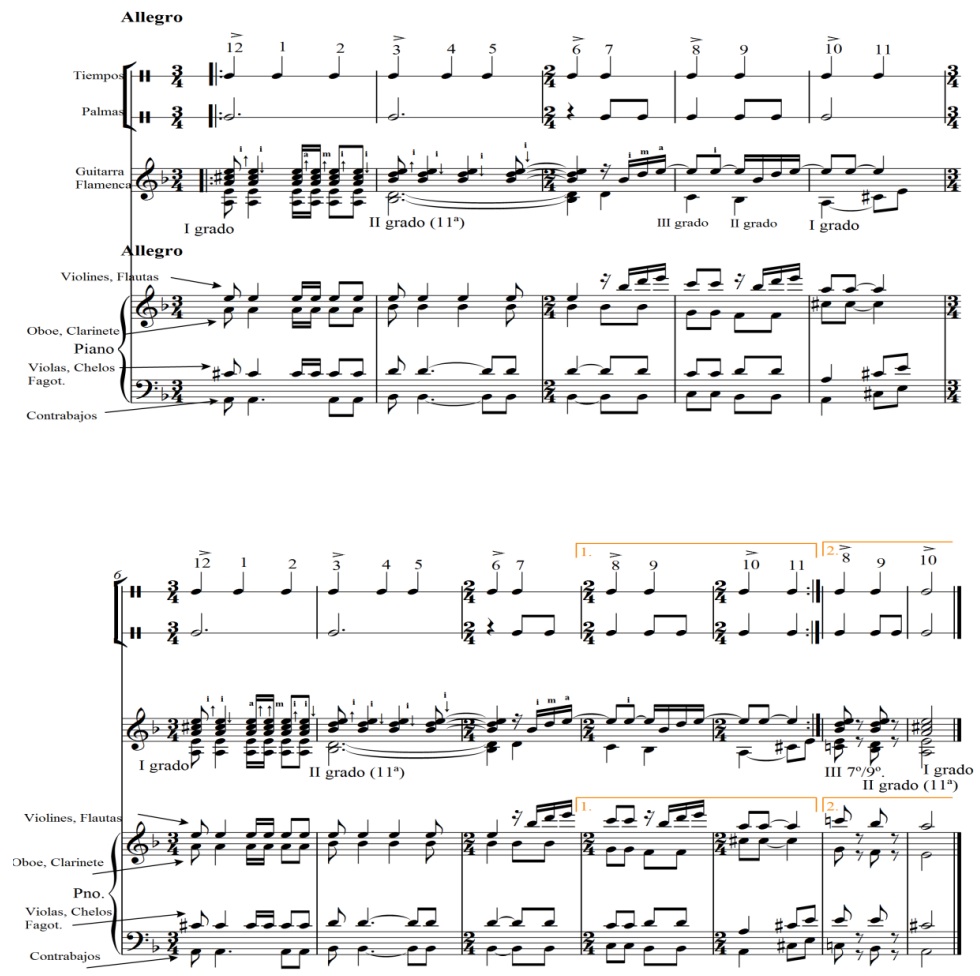

\section{Ejemplo $\mathbf{N}^{0} 7$}

Como ya hemos apuntado anteriormente, la secuencia didáctica que aquí se ha desarrollado también se ha podido aplicar en el contexto de los otros estilos flamencos trabajados en el aula. Además de lo anterior, se han destacado los siguientes aspectos de la metodología igualmente estudiados:

a) Los pasajes de escalas, tan características de la técnica guitarrística flamenca, los cuales se realizan con un "staccato" muy acentuado, lo que es denominado idiomáticamente en guitarra flamenca como "picado", han tenido que ser reinterpretados por los diferentes instrumentos. Esto ha supuesto que las ligaduras de articulación no han sido puestas en la orquestación realizada por el profesor, sino que han sido definidas conjuntamente con el alumnado de las correspondientes secciones instrumentales. El profesor ha reflexionado sobre las características y los propósitos que tiene esta técnica en el lenguaje musical flamenco. El alumnado ha propuesto las diferentes opciones que existían, según la técnica de cada 
instrumento en particular, para indicar las ligaduras en la partitura. Finalmente, se toma una decisión en conjunto con el objetivo de representar de la mejor manera posible el lenguaje flamenco, respetando las características idiomáticas del correspondiente instrumento de tradición clásica.

b) Lo mismo ha ocurrido en relación a las técnicas específicas de la guitarra flamenca denominadas "rasgueo" y "alzapúa", las cuales han tenido que ser reinterpretadas puntualmente por la sección de cuerdas. Aquí, además de las aportaciones del alumnado de dicha sección, el profesor ha mostrado los determinados pasajes de la obra a los respectivos profesores tutores del departamento de cuerda, lo cual ha sido de gran ayuda para la consecución del resultado final.

c) En cuanto a los diferentes acentos y formas de cómo llevar el compás presente en las obras que incluían cante, correspondió al alumnado de tradición flamenca mostrar con ejemplos cómo ellos entienden estos conceptos desde sus propias vivencias. De esta forma, el alumnado de tradición clásica ha podido comprobar y experimentar que tocar "a compás" no es lo mismo que "tener compás". Esto último, según Norberto Torres, lo podemos entender como: "tener bien interiorizada la distribución de acentos de los particulares compases flamencos de amalgama y expresarlos naturalmente" (TORRES, 2005).

\section{La experiencia compartida}

El ejemplo anterior supone ir más allá de lo que podría ser una lectura en conjunto. Supone que el alumnado de tradición clásica está compartiendo los conocimientos musicales previos desde la teoría musical, aplicados ahora en sus respectivos instrumentos. El alumnado de flamenco, a su vez, siente nuevas sonoridades, nuevos timbres, que no han sido tradicionalmente familiares en su cultura musical. Además, la comprensión y la experiencia del alumnado de tradición clásica, referidas a la asimilación del compás de bulería por soleá, han permitido que, llegado un momento en el que han memorizado la parte escrita para su correspondiente instrumento, puedan iniciar variaciones rítmicas y armónicas de manera espontánea, tomando como base lo escrito por el profesor. Se destaca que, llegado este punto, no ha correspondido al profesor indicar o determinar si la variación realizada por el alumno de tradición clásica ha sonado más o menos flamenca; sino que esta valoración la hace el alumnado de tradición flamenca generándose, de esta forma, una discusión constructiva de la experiencia en el aula.

\section{Metodología de la investigación}

\subsection{Participantes}

La muestra sobre la que se efectuó la valoración del aprendizaje estaba compuesta por 47 estudiantes de los cuales 21 corresponden a la asignatura de Conjunto Instrumental Flamenco (9 mujeres y 12 varones), con una edad promedio de $27.7 \pm 6.4$ años, y 26 a la asignatura de Ensemble Flamenco (18 mujeres y 8 varones), con una edad promedio de $21.0 \pm 1.7$ años. Todos los participantes estaban matriculados en di- 
chas asignaturas durante el curso 2015-2016 en el Conservatorio Superior de Música "Rafael Orozco" de Córdoba.

\subsection{Instrumentos}

Se elaboró un instrumento de valoración con 7 ítems destinado a conocer la opinión del estudiante respecto a su propio aprendizaje. Cada ítem fue evaluado con la siguiente escala de 1 a 5, siendo: $1=$ NADA; 2 = POCA; $3=$ ACEPTABLE; $4=$ SIGNIFICATIVA; 5 = MUY SIGNIFIVATIVA.

Los cuatro primeros ítems se refieren a la percepción que tiene el estudiante en relación a los aspectos rítmicos, armónicos, melódicos y técnicos:

\begin{tabular}{|c|l|}
\hline 1 & $\begin{array}{l}\text { Valora la repercusión de los aspectos RÍTIMICOS presentes en la } \\
\text { práctica orquestal flamenca en tu formación instrumental }\end{array}$ \\
\hline 2 & $\begin{array}{l}\text { Valora la repercusión de lso aspectos MELÓDICOS presentes en la } \\
\text { práctica orquestal flamenca en tu formación instrumental }\end{array}$ \\
\hline 3 & $\begin{array}{l}\text { Valora la repercusión de los aspectos ARMÓNICOS presentes en la } \\
\text { práctica orquestal flamenca en tu formación instrumental }\end{array}$ \\
\hline 4 & $\begin{array}{l}\text { Valora la repercusión de la práctica orquestal flamenca en los aspec- } \\
\text { tos TÉCNICOS de tu formación instrumental }\end{array}$ \\
\hline
\end{tabular}

El quinto valora la interacción entre alumnado de diferente tradición.
5
Valora la interacción en el aula con compañeros de otras especiali- dades, en relación a nuevos conocimientos adquiridos (para los de tradición clásica referidos a los de tradición flamenca, para los de tradición flamenca referidos a los de tradición clásica)

El sexto valora la experiencia relacionada con el concierto final de curso en el auditorio del centro.
6
Valora la importancia de la finalización del proceso en concierto pú- blico para tu formación

Y el séptimo valora el grado de satisfacción general con la asignatura.
7
Valora el grado de satisfacción general con la asignatura

También se introdujeron en el cuestionario algunas preguntas para poder filtrar la especialidad de los estudios musicales, la edad y sexo de las y los participantes. 


\subsection{Procedimiento y análisis de datos}

El cuestionario fue administrado el primer lunes de febrero de 2016 durante una hora de clase. Previamente se les informó a los participantes de la voluntariedad y garantías de anonimato. Todo el alumnado convocado decidió participar cumplimentando el cuestionario.

Una vez recogidos los cuestionarios, la información se introdujo en una matriz de datos utilizando el programa estadístico PASW 18. Posteriormente se revisó la matriz de datos y se corrigieron algunos pequeños errores de picado de datos. Finalmente se realizaron análisis de medias y desviaciones típicas.

\section{Resultados}

En la tabla siguiente se recogen los promedios de las valoraciones de los alumnos junto con su correspondiente desviación estándar. Los cuatro primeros ítems corresponden a la evaluación, por parte del alumnado, de los elementos de la ampliación instrumental diseñada para hacer posible la práctica orquestal flamenca de la bulería por soleá y son en los se observa más variabilidad en las valoraciones.

Tabla 1. Puntuaciones promedio y desviaciones estándar resultantes de las valoraciones de los cuestionarios cumplimentados por el alumnado (tanto de tradición clásica como flamenca) de la asignatura de Ensemble Flamenco y Conjunto Instrumental Flamenco (N=47) durante el curso académico 2015-2016.

\begin{tabular}{|l|c|c|c|c|}
\hline \multirow{2}{*}{ Ítem } & \multicolumn{2}{|l|}{ Alumnado tradición clásica (N=26) } & \multicolumn{2}{c|}{ Alumnado tradición flamenca (N=21) } \\
\cline { 2 - 5 } & Promedio & Desviación estándar & Promedio & Desviación estándar \\
\hline 1 & 4.42 & 0.64 & 4.05 & 1.12 \\
\hline 2 & 4.04 & 0.92 & 4.32 & 0.82 \\
\hline 3 & 3.62 & 1.10 & 4.24 & 0.83 \\
\hline 4 & 3.96 & 0.87 & 4.05 & 0.92 \\
\hline 5 & 4.58 & 0.76 & 4.57 & 0.75 \\
\hline 6 & 4.65 & 0.56 & 4.57 & 0.68 \\
\hline 7 & 4.85 & 0.37 & 4.76 & 0.44 \\
\hline
\end{tabular}

La independencia de las dos variables, entendiendo como tales las valoraciones de los alumnos de cada tradición en cada ítem del cuestionario, se ha comprobado mediante el test $\chi^{2}$ para un nivel de confianza del $95 \%$.

Para comprobar si las diferencias que se observan en los promedios de las valoraciones, sobre todo en los cuatro primeros ítems, son estadísticamente significativas, recurriremos a hacer un análisis de la varianza (ANOVA) de los promedios de dichas valoraciones, comparando las varianzas que se dan entre las puntuaciones dentro de cada tradición con la varianza entre ambas tradiciones. Los resultados de este análisis, para un nivel de confianza del $95 \%$, se recogen en la siguiente tabla. 
Tabla 2. Resultados del análisis de varianza (ANOVA) para las valoraciones de cada ítem del cuestionario de las dos tradiciones en estudio (clásica y flamenca) para un nivel de confianza del 95\%.

\begin{tabular}{|l|l|l|l|}
\hline ITEM & $\mathrm{F}$ & $\mathrm{p}$ & SIGNIFICACIÓN \\
\hline 1 & 2,026 & 0,162 & No existen diferencias significativas \\
\hline 2 & 0,323 & 0,572 & No existen diferencias significativas \\
\hline 3 & 4,654 & 0,038 & Existen diferencias significativas \\
\hline 4 & 0,118 & 0,733 & No existen diferencias significativas \\
\hline 5 & 0,002 & 0,964 & No existen diferencias significativas \\
\hline 6 & 0,196 & 0,66 & No existen diferencias significativas \\
\hline 7 & 0,58 & 0,45 & No existen diferencias significativas \\
\hline
\end{tabular}

El único ítem en el que se encuentran diferencias significativas es el número 3, con el que se pretende que el alumnado valore la repercusión de los aspectos armónicos presentes en la práctica orquestal flamenca sobre su formación instrumental. Este ha sido también el ítem que ha obtenido la puntuación más baja del cuestionario, otorgada por el alumnado de tradición clásica. Esto creemos que se debe a que los aspectos armónicos, no implican novedad alguna para alumnos que han estudiado dos cursos de Armonía y otros dos de Análisis Armónico. Sin embargo, este ítem, junto con el correspondiente a los aspectos melódicos presentes en la práctica orquestal, son los mejor valorados por el alumnado de tradición flamenca.

Para el alumnado de tradición clásica, la valoración más alta de estos primeros ítems, aunque ya no existan diferencias significativas entre tradiciones, se corresponde con el $\mathrm{n}^{\mathrm{o}} 1$, relativo a los aspectos rítmicos estudiados. Este ítem presenta una desviación estándar baja, es decir, que ha habido poca dispersión en las respuestas de los citados alumnos. El alumnado de tradición clásica, por lo general, desconoce el ritmo de la bulería por soleá y valora positivamente el trabajo que se ha realizado en el aula encaminado a interiorizar e interpretar dicho ritmo. Paralelamente, este ítem es el que presenta una de las valoraciones más bajas y la mayor desviación estándar para los alumnos de tradición flamenca, ya que el ritmo de este palo flamenco era perfectamente conocido por ellos.

En definitiva, los alumnos valoran la posibilidad de aprender aspectos que, por lo general, no les eran familiares antes de comenzar a cursar las asignaturas de Ensemble Flamenco y Conjunto Instrumental Flamenco.

Las puntuaciones de los tres restantes ítems han sido elevadas y bastante homogéneas en los alumnos de ambas tradiciones, lo cual implica que valoran positivamente la interacción de alumnos provenientes de tradiciones diferentes a la propia, la realización de un concierto conjunto como colofón del curso y la experiencia compartida dentro del desarrollo de la asignatura. 


\section{Discusión y conclusiones}

Compartimos lo expresado por Vilar en cuanto a que "la actividad propia de un conservatorio no debe de ser la música sino la pedagogía de la música" (Vilar, 2000). Sin embargo, creemos que la interpretación musical va mucho más allá del hecho de juntar notas para recrear una melodía, o de un mero ejercicio de psicomotricidad. La interpretación musical supone la comprensión de la obra, de su contexto estético, su cultura, etc. El cúmulo de conocimientos presentes en el curriculum de grado superior de música, lejos de ser un lastre, resulta ser un punto de partida válido y necesario, capaz de impulsar investigaciones que partan desde la teoría de la música o de las propias prácticas artísticas (Ferrando, Pérez López y Muntané, 2006; Zaldivar, $2005,2010)$ para, desde este punto, abordar investigaciones educativas atendiendo a su dimensión didáctica.

La adecuada optimización de las diferentes ampliaciones instrumentales flamencas, entendidas como la suma de conocimientos desde la teoría de la música y la tradición oral presentes en cada estilo flamenco, dará sentido a los significados atribuidos por los estudiantes en relación a las experiencias vividas, como procedimientos de aprendizaje (Rusinek, 2004). También y, en consonancia con lo que apunta Rusinek en su artículo referido a la importancia de los conciertos como finalización del proceso, en nuestro caso se confirma este hecho, ya que los alumnos de ambos perfiles han valorado muy positivamente el ítem $\mathrm{n}^{\circ} 6$ de los cuestionarios, referido a este mismo aspecto.

Podemos concluir que la investigación realizada nos ha permitido profundizar en el conocimiento y comprensión del lenguaje musical flamenco presente en la guitarra flamenca de concierto. La creación y aplicación de las diferentes secuencias didácticas trabajadas en el aula han permitido al docente asumir el rol de profesor e investigador (Stenhouse i, 2003). Esto, sumado a la práctica orquestal en su conjunto, ha permitido la integración del alumnado de tradición flamenca con el alumnado de tradición clásica. Lo anterior también ha resultado ser factor propiciador de un trabajo colaborativo y significativo.

La metodología de trabajo en el aula no solamente se ha basado en la interacción profesor-alumno, sino también en la interacción alumno-alumno. Esto ha ayudado a promover la interdependencia positiva, elemento fundamental en la investigación-acción colaborativa. De esta forma, se ha podido establecer una triangulación que ha repercutido favorablemente en el compromiso del alumnado con los objetivos planteados para cada estilo flamenco, ayudando a tomar conciencia de que las metas propuestas no se pueden conseguir de forma individual, sino que cada miembro del grupo ayuda a promover el aprendizaje en los demás (Johnson y Johnson, 1999).

El hecho de que el alumnado de tradición flamenca no haya mostrado fluidez en la lectura de partituras, a diferencia del alumnado de tradición clásica, no ha resultado ser un problema. Concluimos que el alumnado valora positivamente los aspectos novedosos con respecto a su formación anterior, como se deduce el hecho de que el alumnado de tradición clásica valore por encima del alumnado de tradición flamenca sus aprendizajes en relación a los aspectos rítmicos, mientras que el alumnado de tradición flamenca, en contraste, resalte su aprendizaje avanzado sobre elementos melódicos y armónicos. En definitiva, la experiencia de aprendizaje colaborativo en torno a la orquestación flamenca posibilita a ambos perfiles cultivar con la ayuda del otro aquellos campos de conocimiento que a priori mostraba como más limitados. Se 
infiere que, en base a una misma experiencia colaborativa, al partir de conocimientos previos diferidos, el alumnado de cada tradición despliega procesos cognitivos diferentes. A partir de esta reflexión, se han abordado diferentes estrategias didácticas en el aula encaminadas a crear espacios de interacción, los cuales han promovido la retroalimentación de conocimientos entre alumnado de ambas tradiciones. De esta forma los procesos cognitivos de los aprendizajes previos se han podido conectar con los nuevos aprendizajes en el contexto da la práctica orquestal flamenca, siendo esta conexión un aspecto fundamental para que se produzca un aprendizaje significativo (Ausubel, 1963,1976).

Lo anterior ha generado una nueva realidad de interacción en el aula, en la cual el profesor se ha convertido en un estimulador del aprendizaje, siendo la interacción y la colaboración de los propios alumnos la protagonista de la acción educativa. El profesor-investigador ha podido, como consecuencia de su experiencia en el aula, promover conocimientos prácticos, los cuales le han posibilitado ser sujeto y objeto de la producción de conocimientos (Schön, 1987). Esto ha permitido un ambiente propicio para experimentar nuevos aprendizajes desde un punto de vista cooperativo. Como se vio en el apartado 6, las puntuaciones de los cuestionarios complementados por los alumnos, tanto de tradición clásica como flamenca, difieren de unos a otros en algunos ítems como consecuencia del aprendizaje previo de cada grupo, a saber, los de tradición flamenca que conocen perfectamente los ritmos de los "palos" y los de tradición clásica que dominan los aspectos armónicos de la composición. Aun así, los resultados de los cuestionarios, evidencian que el alumnado valora positivamente su aprendizaje, dados los altos promedios encontrados, siendo el promedio de las valoraciones de todos los ítems superior a 4 (4.33), es decir, que podemos concluir que el alumnado considera que tanto la experiencia del propio aprendizaje como la práctica orquestal flamenca en su formación instrumental han sido significativas.

Lo anterior nos ha permitido conocer la propia percepción del alumno respecto al trabajo en el aula, tanto individual como colectivamente, lo cual es un aspecto importante entendido como factor que nos permite evaluar el aprendizaje significativo (Rivera Muñoz, 2004).

Las notas de clase tomadas después de cada sesión, así como la grabación de los correspondientes encuentros y su posterior interpretación, han permitido al profesor-investigador comprender las experiencias de aprendizaje construidas por el alumnado de ambas tradiciones. El alumnado de grado superior está ya motivado y expectante por experimentar estos nuevos aprendizajes. Este alumnado, que difiere en muchos aspectos, entre ellos el motivacional, del alumnado de instituto o incluso del alumnado de las enseñanzas profesionales de música, necesita, sin embargo, referentes válidos desde su experiencia; necesita asumir como creíbles las herramientas didácticas y compositivas mostradas por el profesor, ya que un estudiante de grado superior de música, por sus propias experiencias previas en las asignaturas de orquesta de cuerdas y/o sinfónica, sabe perfectamente si el desarrollo armónico, melódico y rítmico está bien realizado en una determinada textura. A su vez, el estudiante de tradición flamenca, al escuchar los primeros compases de una orquestación propuesta por el profesor, sabe percibir si ésta conecta o no con el estilo flamenco que él ha experimentado en sus aprendizajes previos desde la tradición oral.

De lo anterior podemos asumir que, la practica orquestal flamenca permite la interacción entre iguales y la transferencia de códigos entre alumnado de tradición clásica con alumnado de tradición flamenca, enriqueciendo el aprendizaje, ayu- 
dándonos a conocer y comprender un nuevo camino metodológico relacionado con la necesaria integración de la especialidad de flamenco en el grado superior de música.

Continuando con lo planteado por Rusinek sobre la necesidad de desarrollar nuevos trabajos colaborativos (Rusinek, 2004), pretendemos que de esta investigación se puedan proyectar nuevos planteamientos colaborativos e interdisciplinares con la finalidad de que, desde las experiencias compartidas, el flamenco se integre como fenómeno musical y cultural en los Conservatorios Superiores de Música. Debemos procurar que el valioso legado que el flamenco nos entrega desde su música y su cultura sea conocimiento válido que nos permita asumir nuevas investigaciones que posibiliten distanciarnos de posturas que pudiesen relegar al flamenco a un gueto epistemológico dentro de los conservatorios. Esta visión colaborativa e interdisciplinar nos permitirá marcar nuevas investigaciones referidas a la música flamenca, desde una dimensión teórica-creativa, didáctica, psicoeducativa e intercultural.

\section{Referencias bibliográficas}

Ausubel, D. P. (1963). The psychology of meaningful verbal learning. New York: Grune \& Stratton.

Ausubel, D. P. (1976). Psicología educativa: un punto de vista cognitivo. México D.F.: Editorial Trillas (traducción al español de Roberto Helier D., de la primera edición de $E d u$ cational psychology: a cognitive view).

Adler, S. (2006). Estudio de la Orquestación. Barcelona: Idea Música.

Carr, W. \& Kemmis, S. (1996). Becoming critical: Education, knowledge and action research. Londres: Falmer Press.

Casals, A., Vilar M. \& Ayats, J. (2008). La Investigación- acción colaborativa; reflexiones metodológicas a partir de su aplicación en un proyecto de música y lengua. Revista Electrónica de Investigación en Educación Musical.

Cera Vera, M. (2010). Sobre el modo flamenco y sus funciones tonales. Revista Musicalia, $8,217-237$.

Elliot, J. (1994). La investigación-acción en educación. Madrid: Ediciones Morata.

Eliott, J. (1991). Estudio del curriculum escolar a través de la investigación interna. Revista Interuniversitaria de Formación del Profesorado, 10, 76-80.

Fernández, L. (2004). Teoría Musical del Flamenco. Madrid: Acordes Concert.

Hernández, F., Pérez López, H. J. \& Gómez Muntané, M. C. (2006). Bases para un debate sobre investigación artística. Colección "Conocimiento Educativo" del Instituto Superior de Formación del Profesorado de la Secretaría General de Educación. España. Madrid: Ministerio de Educación y Ciencia.

Leiva, M. A. \& Mates, E. M. (1998). Importancia de la educación musical en la formación integral del hombre. Música y Educación, 33, 27-40.

Lewin, K. (1946). Action Research and Minority Problems. Journal of Social Issues. 4.34-46. (traducción de María cristina Salazar, Universidad Nacional de Colombia, Bogotá, 1990).

Manrique, W. (1997). La investigación-acción y el mejoramiento de la calidad del docente en el aula. Educare, 1(1), 39-59.

Miller, B. A. (1996). Integrating elementary general music: A collaborative action research study. Bulletin of Council for Research in Music Education, 130, 100-115. 
Piston, W. (1984). Orquestación. Madrid: Real Musical.

Ramírez, C. (2006). Música, lenguaje y educación. La comunicación humana a través de la música en el proceso educativo. Valencia: Tirant lo Blanch.

Rivera Muñoz, L. (2004). El aprendizaje significativo y la evaluación de los aprendizajes. Revista de Investigación Educativa, 14, 47-52.

Rusinek, G. (2004). Aprendizaje musical significativo. Revista Electrónica Complutense de Investigación en Educación Musical, 1 (5), 1 - 16 [http://www.ucm.es/info/reciem/index. $\mathrm{htm}]$

Schön, D. (1987). Educating the reflective practitioner. San Francisco: Jossey - Bass.

Stenhouse, L. (1993). La investigación como base de la enseñanza. Madrid: Ediciones Morata.

Stenhouse, L. (2003). Investigación y desarrollo del curriculum. Madrid: Ediciones Morata.

Torres, N. (2005). Historia de la guitarra flamenca. El surco, el ritmo y el compás. Córdoba: Editorial Almuzara. 24 - 25.

Vilar, J. M. (2000). Mesa redonda: “Investigación aplicada” Investigación-acción y currículo oculto en la enseñanza obligatoria. Revista electrónica LEEME, 5.

Zaldívar Gracia, A. (2005). Las enseñanzas musicales y el nuevo Espacio Europeo de Educación Superior: el reto de un marco organizativo adecuado y la necesidad de la investigación creativa y "performativa". Revista Interuniversitaria de Formación del Profesorado, 52. 95-122.

Zaldívar Gracia, A. (2010). Investigación desde la práctica artística. Libro de actas I Congreso de Investigación en Música. Valencia. 